University of Warwick institutional repository: http://go.warwick.ac.uk/wrap This paper is made available online in accordance with publisher policies. Please scroll down to view the document itself. Please refer to the repository record for this item and our policy information available from the repository home page for further information.

To see the final version of this paper please visit the publisher's website. Access to the published version may require a subscription.

Author(s): Y. S. Tang, C. D. W. Wilkinson, C. M. Sotomayor Torres, D. W. Smith, T. E. Whall, and E. H. C. Parker

Article Title: Optical properties of $\mathrm{Si} / \mathrm{Si}_{0.87} \mathrm{Ge}_{0.13}$ multiple quantum well wires

Year of publication: 1993

Link to published version: http://dx.doi.org/ 10.1063/1.109984

Publisher statement: none 


\title{
Optical properties of $\mathrm{Si} / \mathrm{Si}_{0.87} \mathrm{Ge}_{0.13}$ multiple quantum well wires
}

\author{
Y. S. Tang, C. D. W. Wilkinson, and C. M. Sotomayor Torres \\ Nanoelectronics Research Centre, Department of Electronics and Electrical Engineering, \\ University of Glasgow, Glasgow G12 8QQ United Kingdom \\ D. W. Smith, T. E. Whall, and E. H. C. Parker \\ Department of Physics, University of Warwick, Coventry CV4 7AL, United Kingdom
}

(Received 15 December 1992; accepted for publication 11 May 1993)

\begin{abstract}
Nanometer-scale wires cut into a $\mathrm{Si} / \mathrm{Si}_{0.87} \mathrm{Ge}_{0.13}$ multiple quantum well structure were fabricated and characterized by using photoluminescence and photoreflectance at temperatures between 4 and $20 \mathrm{~K}$. It was found that, in addition to a low-energy broadband emission at around $0.8 \mathrm{eV}$ and other features normally observable in photoluminescence measurements, fabrication process induced strain relaxation and enhanced electron-hole droplets emission together with a new feature at $1.131 \mathrm{eV}$ at $4 \mathrm{~K}$ were observed. The latter was further identified as a transition related to impurities located at the $\mathrm{Si} / \mathrm{Si}_{0.87} \mathrm{Ge}_{0.13}$ heterointerfaces.
\end{abstract}

The physics of nanostructures, such as quantum wires and dots, based on quasi-two-dimensional (2D) structures, such as modulation doped 2D electron gas, superlattices (SLs), and multiple quantum wells (MQWs), has been receiving worldwide attention ${ }^{1}$ in the last few years due to its promising device applications. $\mathrm{Si} / \mathrm{Si}_{1-x} \mathrm{Ge}_{x}$ heterostructures, as one of the recently emerged new materials, were also widely investigated ${ }^{2-7}$ with most of the emphasis on optical properties of $2 \mathrm{D}$ structures. With the development of nanofabrication techniques, e.g., electron beam lithography and reactive ion etching, features with minimum dimension down to the sub-50-nm range can be defined. ${ }^{8}$ Both the studies of sidewall damage induced during dry etching, which has been proved to be little for the $\mathrm{Si}$ / $\mathrm{Si}_{1-x} \mathrm{Ge}_{x}$ system compared to the $\mathrm{GaAs} / \mathrm{Al}_{1-x} \mathrm{Ga}_{x}$ As system, ${ }^{9}$ and the properties of $\mathrm{Si} / \mathrm{Si}_{1-x} \mathrm{Ge}_{x}$ nanostructures ${ }^{10,11}$ have been recently reported. The studies on the optical properties of nanostructured wires based on a modulation doped $p^{+}-\mathrm{Si} / \mathrm{SiGe} 2 \mathrm{D}$ hole gas indicate that a strain relaxation process occurs during the wire fabrication and a blue shift of quantum confined transitions with reducing lateral dimensions down to below about $40 \mathrm{~nm}$ can be observed. ${ }^{10,11}$ In this letter, we report a study on the optical properties of a series of $\mathrm{Si} / \mathrm{Si}_{1-x} \mathrm{Ge}_{x} \mathrm{MQW}$ wires. In addition to the features similar to those reported on the $2 \mathrm{D}$ hole gas wires, an enhanced electron-hole droplets (EHD) emission and a new feature related to the $\mathrm{Si} / \mathrm{Si}_{1-x} \mathrm{Ge}_{x}$ heterointerfaces have been observed in the MQW wires. Possible origin of the new feature is also discussed.

The original material was grown by molecular-beam epitaxy (MBE) on a (100) oriented $n-\operatorname{Si}(\rho \sim 20 \Omega \mathrm{cm})$ substrate heated to $550^{\circ} \mathrm{C}$. The evaporation of $\mathrm{Si}$ and $\mathrm{Ge}$ using an electron beam yields a growth rate of $0.1 \mathrm{~nm} / \mathrm{s}$. The MBE sample has 30 periods of $\mathrm{Si} / \mathrm{Si}_{0.87} \mathrm{Ge}_{0.13} \mathrm{MQWs}$ with the well and barrier widths of 8 and $21 \mathrm{~nm}$, respectively, grown on a $200 \mathrm{~nm} \mathrm{Si} \mathrm{buffer} \mathrm{layer.} \mathrm{They} \mathrm{were} \mathrm{first}$ scribed into a series of $5 \mathrm{~mm} \times 5 \mathrm{~mm}$ chips, and then cleaned in methanol, acetone, and isopropanol for $5 \mathrm{~min}$ in an ultrasonic bath followed by rinsing in deionized water and finally blown dry in nitrogen gas. After spinning a double layer of PMMA $^{8}$ as the electron beam resist, the samples were then patterned with wires of different sizes using electron beam lithography and lift-off of the resist.

The reactive ion etching was performed on a planar reaction chamber system operated at an rf frequency of 13.56 MHz. The etching gas was $99.99 \%$ pure $\mathrm{SiCl}_{4}$. By using the optimized etching parameters described elsewhere, ${ }^{9}$ we obtained a series of $2.5-\mu \mathrm{m}$-long wires with their widths between 40 and $500 \mathrm{~nm}$ and pitch size of 750 $\mathrm{nm}$. The etched depth was kept at about $0.6 \mu \mathrm{m}$, i.e., half of the MQW layers was cut into wires, so that we can have simultaneous signals from both the wires and the remaining MQWs, which serve as a control sample, in optical measurements.

The photoluminescence (PL) was measured at temperatures between 4 and $20 \mathrm{~K}$ on a standard cryostat system, using an $\mathrm{Ar}^{+}$ion laser $(488 \mathrm{~nm})$ with a power density variable between 0.1 and $50 \mathrm{~W} / \mathrm{cm}^{2}$. The luminescence signal was detected by a liquid $\mathrm{N}_{2}$ cooled $\mathrm{Ge}$ detector through a Jobin-Yvon THR 1000 monochromator.

The photoreflectance (PR) measurements were performed on a setup system similar to that described elsewhere. ${ }^{12}$ Both the excitation beam and the modulation beam were monochromatic light sources from $70 \mathrm{~W}$ tungsten-halogen lamps, and the beam intensity was kept at a low level to guarantee that the measurements were performed in the low field modulation limit. ${ }^{13}$ The signal was detected by either a $\mathrm{Si} p-i-n$ diode or a liquid $\mathrm{N}_{2}$ cooled Ge detector.

Figure 1 shows the PL spectra of a MQW sample (b) and four wire samples (c)-(f) at $4 \mathrm{~K}$. For comparison, a PL spectrum from a deep etched sample (a) is also plotted in Fig. 1, from which all the epigrown layers were removed. The PL spectra were obtained under a constant illuminated area on different samples, i.e., more etched areas are exposed to the laser beam during the measurements with reducing wire width. It can be seen that ${ }^{2,3}$ peaks 1 and 4 are from the substrate, which are the bound exciton nophonon line $\left(\mathrm{BE}_{\mathrm{Si}}\right)$ and the TO phonon assisted bound exciton $\left(\mathrm{BE}_{\mathrm{Si}}^{\mathrm{TO}}\right.$ ) line respectively; peaks 3 and 5 are TO phonon assisted free exciton $\left(F E_{\mathrm{Si}}^{\mathrm{TO}}\right.$ ) line and the EHD emission, about $12 \mathrm{meV}$ below the $\mathrm{BE} \mathrm{Si}_{\mathrm{Si}}^{\mathrm{TO}}$ line, related to the Si buffer layer or the Si layers in the MQWs. It is obvious that even with less illuminated areas on the wires, an en- 


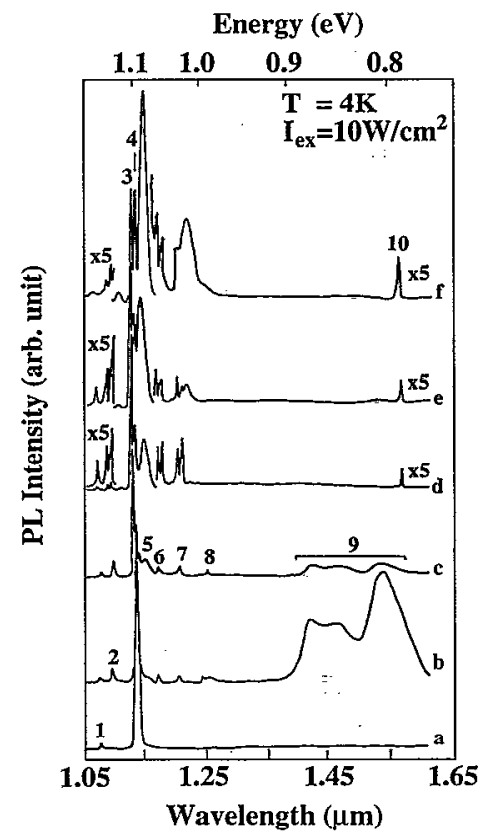

FIG. 1. PL spectra of different samples at $4 \mathrm{~K}$ with the excitation power density of $10 \mathrm{~W} / \mathrm{cm}^{2}$. The peaks are marked by the following numbers: (1) $\mathrm{BE}_{\mathrm{Si}}$; (2) $X_{I}$; (3) $\mathrm{FE}_{\mathrm{Si}}^{\mathrm{TO}}$; (4) $\mathrm{BE}_{\mathrm{Si}}^{\mathrm{TO}}$; (5) $\mathrm{EHD}$; (6) $X_{\mathrm{Si}-\mathrm{Ne}}^{\mathrm{NP}}$ (7) $\mathrm{SiGe}$ related; (8) $X_{\mathrm{Si}-\mathrm{Ge}}^{\mathrm{TO}}$; (9) strain related; (10) defects. (a) deep etched sample where all epitaxial layers are removed away; (b) multiple quantum wells; (c) $2.5 \mu \mathrm{m} \times 500 \mathrm{~nm}$ wires; (d) $2.5 \mu \mathrm{m} \times 200 \mathrm{~nm}$ wires; (e) 2.5 $\mu \mathrm{m} \times 100 \mathrm{~nm}$ wires; (f) $2.5 \mu \mathrm{m} \times 40 \mathrm{~nm}$ wires.

hanced luminescence of EHD possibly due to the increasing surface roughness introduced during the wire fabrication process, which improves the formation of the nucleation of the droplets, leading to stronger PL in the cxpcriments ${ }^{14}$ is still obscrved under the same excitation condition. This behavior is similar to that reported before ${ }^{15}$ where alloy disorder accelerates the formation of nucleation of droplets in $\mathrm{Al}_{x} \mathrm{Ga}_{1-x}$ As. In addition to those peaks discussed above, other excitonic features (6-8) related to the SiGe layers in the MQWs are also observed, which are no-phonon and TO phonon assisted PL lines. The low-energy broadband transition (9) at $\sim 0.8 \mathrm{eV}$ existing in both the MQWs and a 500-nm-wide wire sample might be related to strain or dislocations, and a new feature $X_{I}$ (2) located between the no phonon and TO phonon assisted Si PL lines emerges in both the MQWs and the wire samples. It must be from the $\mathrm{Si}$ buffer layer on the $\mathrm{Si}$ layers in the MQWs due to its position. When the wire width is reduced to about $200 \mathrm{~nm}$, broadband 9 disappears, a new defects related peak (10) emerges at $0.76 \mathrm{eV}$, and the peaks related to the SiGe layers $(6,7)$ split into two, indicating a strain relaxation process occurred during the dry etching. While the splitting of peak 2 suggests that it is from the Si layers in the MQWs due to the fact that only half of the MQWs were etched away, no double structure should be seen if it was from the Si buffer layer. The blue shift of the split peak is due to strain relaxation in the $\mathrm{Si}$ layers as half of the MQWs was etched away.

The origin of band 9 in Fig. 1 deserves more study, but a possible explanation for the intensity drop and disappear-

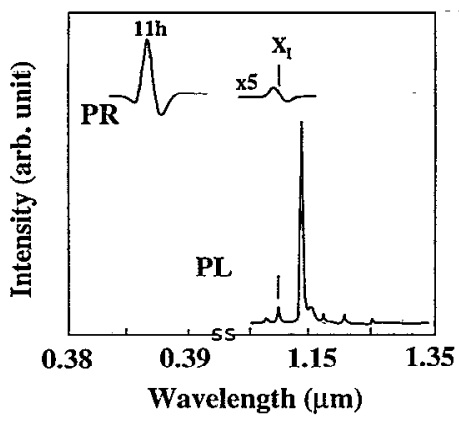

FIG. 2. Comparison of PR and PL spectra from a 500-nm-wide wire sample at $4 \mathrm{~K}$.

ance of it with reducing wire width is that this band may come from strain in the epilayers. As half of the MQWs were etched into wide wires, the strain will partially be relaxed, causing a decrease in its PL intensity. As the wires narrow further, more areas probed in the PL experiments are in the dry etched region and somehow damaged. Although half of the MQWs remain untouched, the surface damage and the total relaxation of strain in the MQW wires may extinguish any optical emission from the samples.

To identify the origin of the new feature $X_{I}$, we performed PR measurements on the samples. As shown in Fig. 2, in addition to the high-energy transition at 3.2 $\mathrm{eV},{ }^{10,11}$ a low-energy transition corresponding to $X_{I}$ in PL appears which must be direct band-gap transition. As we know, the origins of a pseudodirect band gap can be due to either Brillouin zone folding in a SL or defects/impurities at the heterointerfaces. ${ }^{4,5}$ In our case, both the well and barrier layers are very thick, i.e., they are not SLs but MQWs, which eliminates the possibility of the zone folding origin and leaves the possible origin to defects/impurities at the heterointerfaces.

To understand further the nature of $X_{I}$, we have performed both temperature- and excitation power-dependent measurements on these samples. Shown in Fig. 3 is the

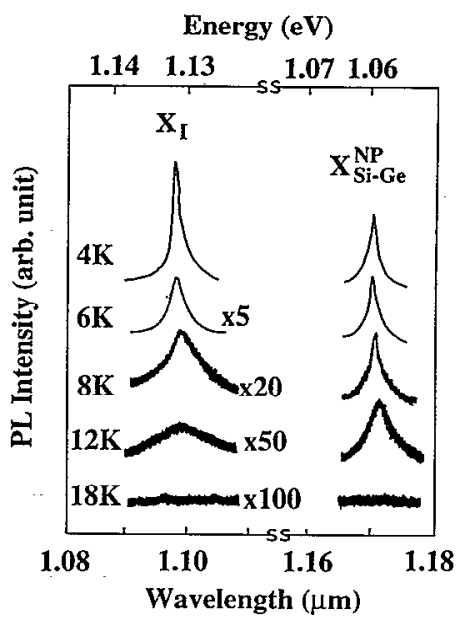

FIG. 3. Temperature dependence of PL spectra from a 500-nm-wide wire sample. The excitation power density is about $10 \mathrm{~W} / \mathrm{cm}^{2}$. 


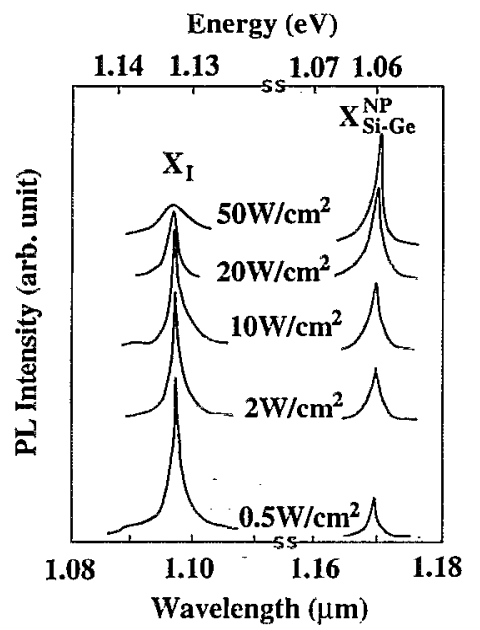

FIG. 4. Excitation power density dependence of PL spectra from the 500 -nm-wide wires at $4 \mathrm{~K}$.

temperature-dependent PL of $X_{I}$ from a 500-nm-wide wire sample with the excitation power of about $10 \mathrm{~W} / \mathrm{cm}^{2}$, where the intensity of $X_{I}$ has been normalized to the intensity of $X_{\mathrm{Si} \text {-Ge }}^{\mathrm{NP}}$ (peak 6 in Fig. 1). With the increase of temperature from 4 to $8 \mathrm{~K}$, the PL intensity of $X_{I}$ gradually drops to zero as compared to the intrinsic PL peak, i.e., $X_{\text {Si-Ge }}^{\mathrm{NP}}$. At a temperature of about $18 \mathrm{~K}$, no PL signal is observable. The exact reason for the quenching of PL signals from $\mathrm{Si} / \mathrm{SiGe}$ heterostructures at higher temperatures is not clear, but similar rapid decreasing line intensities with increasing temperature were also observed by other researchers where the effect was explained by the dissociation of excitons from the binding centers. ${ }^{6}$

Figure 4 shows the PL spectra of the 500-nm-wide wires at $4 \mathrm{~K}$ with varying excitation power. It can be seen that with the increase of the optical pumping power, the intense $X_{I}$ gradually decreases whereas $X_{\mathrm{Si}-\mathrm{Ge}}^{\mathrm{NP}}$ increases its intensity, indicating that $X_{I}$ is a transition related to impurities. It is reasonable to propose that $X_{I}$ arises from impurity states of unknown origin located at or in Si layers close to the heterointerfaces. The feature in lowtemperature PL spectra may be from an impurity to band transition which is also observable in the PR measurements.

In conclusion, we have presented for the first time a study on the optical properties of a dry etched free standing $\mathrm{Si} / \mathrm{Si}_{1-x} \mathrm{Ge}_{x} \mathrm{MQW}$ wire system. The results show that, in addition to the luminescence due to a strain relaxation process arising from the etching, an enhanced emission from EHD in the wires with reduced lateral dimension and a new feature probably due to impurity states located at the $\mathrm{Si} / \mathrm{SiGe}$ heterointerfaces have been detected.

${ }^{1}$ For example, Nanostructure Physics and Fabrication, edited by M. A. Reed and W. R. Kirk (Academic, San Diego, 1989).

${ }^{2}$ D. J. Robbins, L. T. Canham, S. J. Barnett, A. D. Pitt, and P. Calcott, J. Appl. Phys. 71, 1407 (1992).

${ }^{3}$ L. Vescan, A. Hartmann, K. Schmidt, Ch. Dieker, H. Luth, and W. Jager, Appl. Phys. Lett. 60, 2183 (1992).

${ }^{4}$ S. Froyen, D. M. Wood, and A. Zunger, Phys. Rev. B 36, 4547 (1987).

${ }^{5}$ I. Morrison, M. Jaros and K. B. Wong, Phys. Rev. B 35, 9693 (1987); G. Abstreiter, in Physics of Semiconductors, edited by X. Xie and K. Huang (World Scientific, Singapore, 1992).

${ }^{6}$ T. D. Steiner, R. L. Hengehold, Y. K. Yeo, D. J. Godbey, P. E. Thompson, and G. S. Pomrenke, J. Vac. Sci. Technol. B 10, 924 (1992).

${ }^{7}$ H. Presting, H. Kibbel, M. Jaros, R. M. Turton, U. Menczigar, G. Abstreiter, and H. G. Grimmeiss, Semicon. Sci. Technol. 7, 1127 (1992).

${ }^{8}$ Y. S. Tang, R. Cheung, and C. D. W. Wilkinson, Electron. Lett. 26, 2389 (1990).

${ }^{9}$ Y. S. Tang, C. D. W. Wilkinson, D. W. Smith, T. E. Whall, and E. H. C. Parker (unpublished).

${ }^{10}$ Y. S. Tang, C. D. W. Wilkinson, C. M. Sotomayor Torres, D. W. Smith, T. E. Whall, and E. H. C. Parker, Superlattices and Microstructures 12, 535 (1992).

${ }^{11}$ Y. S. Tang, C. D. W. Wilkinson, C. M. Sotomayor Torres, D. W. Smith, T. E. Whall, and E. H. C. Parker, Solid State Commun. 85, 199 (1993).

${ }^{12}$ Y. S. Tang, B. S. Wang, D. S. Jiang, W. H. Zhuang, and J. B. Liang, Solid State Commun. 63, 793 (1987).

${ }^{13}$ Y. S. Tang, Phys. Status Solidi B 154, K187 (1990).

${ }^{14}$ Y. S. Tang, C. D. W. Wilkinson, C. M. Sotomayor Torres, D. W. Smith, T. E. Whall, and E. H. C. Parker (unpublished).

${ }^{15}$ H. Kalt, K. Reimann, W. W. Ruehle, M. Rinker, and E. Bauser, Phys. Rev. B 42, 7058 (1990). 